\title{
La sociedad del conocimiento: El orden del cambio*
}

\author{
Emilio Lamo de Espinosa' \\ Universidad Complutense \\ Instituto Universitario Ortega y Gasset
}

\section{Introducción}

¿Qué es la técnica o la tecnología? ¿Cómo se produce? Sobre todo, ¿por qué innovamos, cómo lo hacemos, quién lo hace? Pretendo abordar el tema de un modo distinto al usual pues, como señaló Whitehead hace ya años ${ }^{2}$, el conocimiento científico-técnico nos rodea de tal modo, es ya parte tan natural de nuestra existencia, que perdemos la dimensión real de su importancia. Para captarla es pues necesario salir del marco de la actual sociedad del conocimiento, e incluso en alguna medida del marco la civilización occidental, para vislumbrar su proceso de desarrollo, su inmenso crecimiento actual. Pues a mi entender la cuestión importante no es tanto la de los efectos concretos de esta o aquella tecnología, de la microelectrónica, la ingeniería genética o los nuevos materiales, por citar algunas, sino el efecto agregado de la tecnología misma, lo que significa para una civilización la producción sistemática de conocimientos.

* En esta edición publicamos integramente el presente artículo,s subsanando así los errores del número anterior (N. del E.) 


\section{Una mirada a vista de pájaro: los tres saltos adelante}

Para ello, hagamos un experimento mental: tratemos de retroceder en la historia, de recular y distanciarnos para tener perspectiva, y desde ahí, lancemos una mirada a vista de pájaro sobre la evolución de los conocimientos en la historia de la humanidad, al modo, por ej. en que lo hace Jared Diamond en su excelente libro Armas, gérmenes y acero ${ }^{3}$ o al modo en que lo hizo ya hace años Gordon Childe en La revolución del neolítico ${ }^{4}$. Y lo que descubrimos es un pauta clara de dinámica ascendente, constante pero no continua en la que hay tres grandes saltos adelante, tres momentos calientes en los que el grado de nuestros conocimientos se eleva considerablemente esto lo podemos comprobar simplemente analizando el cambio social, consecuencia casi siempre de innovaciones tecnológicas.

Efectivamente, en el 40.000 A.C. se vivía igual que en el 9.000 A.C., pero de modo muy distinto a como se vivía en el 3.000; es el primer salto adelante, la revolución del neolítico que da lugar a las primeras ciudades (Jericó), los primeros Estados y los primeros Imperios. Pero del 1.000 A.C. al 1.000 d.c., incluso al 1.400, de Egipto o Mesopotamia al medioevo, las diferencias son pequeñas y el modo de vida continuará estable hasta la revolución de la ciencia en la Inglaterra y Holanda del siglo XVII y la posterior Revolución Industrial. Por ello, la vida cotidiana en el XVII, incluso el XVIII, no era muy diferente de la del siglo I, pero es muy distinta a como se vivía a mediados del XX. Es el impacto de la revolución científica clásica, la primera, que por cierto afecta sólo a Europa y es la causa directa de su expansión hasta llegar a ser, hoy en día, la primera civilización universal. Finalmente, hace setenta años, nuestros padres (no digamos nuestros abuelos), vivían igual que a mediados del XIX, con pequeñas variaciones que van incorporando a sus vidas. Pero ellos vivieron de modo muy distinto a como se vive hoy. Estamos de lleno en la actual revolución científico-técnica, la segunda gran revolución científica.

Son tres grandes saltos adelante - la revolución neolítica, la primera revolución científica del XVII y la actual revolución científico-técnica, que marcan tres inmensas fronteras en el desarrollo de los conocimientos y, como consecuencia, en las formas de vida. Sin duda alli donde esos puntos de inflexión son más visibles es en la dinámica demográfica de la humanidad. Se estima que hacia el año 10.000 a.C. había no más de 5 millones de hombres en el planeta. Tras la revolu- 
ción del neolítico, y para el año cero de nuestra era, el número había crecido a unos 250 millones. Y para el año 2.010 seremos probablemente unos 7.000 millones. Pero podríamos poner otros muchos ejemplos. Así, si lo midiéramos en energía disponible por habitante y año veríamos como se produce también un salto inmenso antes y después de esas tres fronteras: de $0,5 \mathrm{cv}$ en la antigüedad, a 1,6 en 1870 y más de 15 hoy. Podíamos medirlo de otros modos: en velocidad de transporte $(65 \mathrm{Km}$./día en la antigüedad; 325 hacia 1870 ; unos 60.000 hoy); en potencia explosiva $(0,5 \mathrm{Kg}$. de TNT en la antigüedad; 500 en 1870; 10 elevado a 8 Toneladas de TNT hoy). Y por supuesto, en la esperanza de vida: 22 años en la antigüedad; 45 en 1870; 68 hoy. Y podría continuar con cientos de ejemplos que, en todo caso, nos muestran que si en algún campo podemos hablar de progreso de la humanidad, si la teoría clásica del progreso (Turgot, Condorcet) puede predicarse de algo, ese algo es, sin duda el progreso de los conocimientos. Podemos dudar del progreso moral de la humanidad y podemos preguntarnos si el progreso estético tiene o no sentido, pero no podemos dudar del progreso cognitivo.

Pero, ¿a que se debe ese progreso? ¿Qué ha impulsado la innovación y el descubrimiento? Veamos muy rápidamente la causa de los dos primeros saltos.

La revolución neolítica - como la denominó Gordon Childe- se inicia hacia el 10.000 a.c. con la aparición de la agricultura, la domesticación de los animales, la cerámica y la alfarería, seguida del uso del bronce y el hierro y que da lugar a las primeras ciudades y después los primeros Imperios históricos. Es el paso de la prehistoria a la historia de la humanidad. Pronto aparecerá la escritura y antes los diversos alfabetos.

Pues bien, ¿que fue lo que originó esa oleada inmensa, gigantesca de innovaciones que va a cambiar por completo la historia de la humanidad? Desde luego no fue un inventor pues sabemos que ocurrió en varios lugares y en distintos momentos: el creciente fértil, Egipto, India y China, más tarde en meso América y, según parece, en el este de los actuales USA e incluso probablemente en Nueva Guinea (según señala Diamond). Fue, como agudamente observo Ortega y Gasset en un texto excelente, la Meditación sobre la técnicas ( $\mathrm{y}$, sin saberlo, refuerza Diamond) resultado del puro azar, lo que llamaba la técnica

La sociedod del conocimiento: El orden del cambio 
del azar. El contacto continuo de grupos humanos con un entorno favorable les habituó espontanea e inconscientemente a manipular semillas o mamíferos, a vivir con ellos. Y ese espontaneo "vivir con" dio lugar a un aprendizaje, a un conocimiento natural y espontáneo, de modo que nadie se planteó el problema de la innovación, nadie quiso innovar. Por eso, Diamond ofrece una explicación convincente de los orígenes de la civilización — de la revolución del neolíticocomo simple proceso evolutivo.

Es más sabemos por otros estudios que este tipo de sociedades tradicionales y estables, recelan de las novedades y las innovaciones; yo las he llamado (con Malinowski) neófobas ${ }^{6}$. Lo que ocurre es que en ellas, por así decirlo, la evolución natural adaptativa a un nicho ecológico determinado da lugar a una serie de conocimientos que forman parte natural de su cultura. El bábitat genera hábitos de todo tipo que incorporan conocimiento del entorno ( $y$ estoy abusando, conscientemente, de categorías puestas en circulación por Bourdieu). No hay propiamente ciencia sino una cultura que incorpora saberes de modo natural y evolutivo. Hay saber, y sobre todo "saber hacer", más que conocer. En ellas la cultura es la ciencia, la cultura incorpora de modo espontáneo, natural e inconsciente, un buen arsenal de conocimientos científicos que les permiten lidiar con las incertidumbres del entorno, del bábitat. Por ello, como decía Ortega, el primitivo no sabe que puede inventar e incluso ignora su propia técnica' ${ }^{7}$. Pero, por supuesto, la tiene.

El segundo gran salto adelante es la revolución científica del XVII con la que pasamos - siguiendo de nuevo a Ortega - desde la técnica del azar a la técnica del técnico. El hombre moderno, antes de inventar sabe que puede inventar; esto equivale a que antes de tener una técnica tiene la técnica ${ }^{8}$. Pues lo importante, lo que fue crucial de aquella revolución científica no es que se saben muchas cosas sino que se sabe la más importante de todas: se sabe que se puede saber más y, sobre todo, cómo se puede saber más. Lo específico de la revolución científica -y lo específico de la civilización occidental- es que se ha descubierto cómo descubrir, y como descubrir cualquier cosa, como descubrir sistemática y constantemente. Y esa es la gran innovación: aprender a aprender, descubrir cómo descubrir, cómo innovar.

Por eso los comienzos de la ciencia moderna debemos situarlos en el descubrimiento del método científico pues es el método, es decir, el 
modo, el procedimiento, la máquina de adquirir nuevos conocimientos, es la esencia de ese aprender a aprender. Repito que, lo importante de la revolución científica del XVII no fue la adquisición de nuevos conocimientos sino la adquisición del conocimiento básico: cómo conocer. Y cómo conocer es el método. No es ciertamente casual que en poco más de tres lustros aparezcan los dos grandes tratados del método científico: el Novum Organum del inglés Bacon (1620), que descubre el método experimental, inductivo, sintético, que va de lo particular a lo general; y el Discurso del método (1637) del francés Descartes, que descubre el método lógico-matemático, deductivo, analítico, que va de los general a lo particular;. Por supuesto la ciencia se hace combinando los dos, mezclando inducción y deducción y esa, que había sido la aportación pionera de Galileo, será formalizada por el gran científico Newton en los Principia, sin duda una de las cabezas mas brillantes de la historia de la humanidad, y formalizado por Kant en la Crítica de la razón teórica.

Y esta idea, la de que la clave de la ciencia no radica en sus descubrimientos sino en el descubrimiento de cómo descubrir $\mathrm{y}$, por lo tanto, de que se puede descubrir todo, no es una idea mía pues fue expresada con toda claridad tanto por Bacon como por Descartes. Los dos sabían perfectamente lo que estaban haciendo. Lo más excelso, dice Bacon, es descubrir aquello por lo que todo lo demás puede ser descubierto con facilidad. En cuando a método - señala Descartes en el Discurso del método- entiendo por ello reglas ciertas y fáciles cuya exacta observancia permite que nadie tome nunca por verdadero nada que sea falso y que llegue mediante un acrecentamiento gradual y continuo de la ciencia, al verdadero conocimiento de todo lo que sea capaz de conocer.

Y por ello podemos decir que a partir del XVII la humanidad no innova azarosamente, casi a pesar suyo, como hacía con anterioridad, sino que innova voluntaria y conscientemente. El propio Bacon, gran visionario, diseñó el primer laboratorio o universidad moderna, investigadora (precedente claro de Humboldt), a la que llamó la Casa de Salomón, la casa de la sabiduría, y que fue el antecedente directo de la primera sociedad científica, la Royal Society inglesa. Pronto Luis XIV en Francia, Catalina la Grande en Rusia, Federico de Prusia o Carlos III en España, seguirán ese mismo camino mientras, como sabemos, las universidades vivían por completo al margen de la gran revolución científica encerradas en el escolasticismo, el silogismo y las citas de 
autoridades, el viejo órgano o método contra el que se rebelaba el experimentalista Bacon. Pero se habia descubierto la máquina de producir descubrimientos y con ello comenzará la andadura de la ciencia y la técnica modernas.

$Y$ asi, si el XVII fue el siglo de la astronomia y la fisica, el XVIII, con Lavoisier, será el de la quimica, y el XIX, con Darwin, será la biologia la que sufrira su revolución basta los comienzos del $X X$ en que Planck y Einstein renovaron de nuevo la física. Todo ello a través de un camino ascendente e indiscutiblemente progresivo. Nunca fue más cierta la vieja idea con la que Newton, ese beredero de los magos babilonios (como lo caracterizó Keynes) trató de minimizar su genialidad: si llegué a ver tan lejos, es porque pude alzarme sobre hombros de gigantes'.

Además, tan pronto se descubre la técnica, esta se aplica a las artes industriales y a la producción, mecanizando instrumentos y procesos, cuyo origen podemos datarlo en 1776 , fecha de la maquina de vapor de Watt (pero también de La riqueza de las naciones de Smith). Es la invención de la tecnología sustentada en la máquina que sustituye al músculo. Poco antes, en 1747, se fundaba la primera escuela de ingeniería l'Ecôle des Ponts et Chaussées. El gran libro de esa primera revolución es la Enciclopedia (1751-1772). Y con ello comienza la revolución de la productividad, que se potenciará, con la segunda revolución industrial, desde finales de siglo, cuando el conocimiento se aplica a mecanizar el trabajo mismo, los modos de organizar el trabajo: es el taylorismo (1881) y el fordismo, que da lugar a la revolución de la productividad. Y efectivamente, el resultado de esa primera revolución tecnológica es que la productividad comenzó a crecer al ritmo del 3,5 o 4\% anual, doblándose cada 18 años (y retengamos esa cifra). Desde los tiempos de Taylor se estima que la productividad se ha multiplicado por 50 , multiplicando al tiempo la riqueza disponible y el bienestar.

\section{La sociedad del conocimiento: sus causas}

No obstante la ciencia se encontraba con numerosas resistencias. Ideológicas, por supuesto, y recordemos a Galileo. Pero sobre todo, era la actividad de pioneros, individuos aislados sin más recursos que los que ellos mismos ponían (como Darwin) o dependiendo de algún mecenazgo más o menos arbitrario. $\mathrm{Y}$ esto de los recursos nos lleva directamente a la clave de la actual revolución científico-técnica. 
Efectivamente, ya el propio Bacon distinguia en la ciencia tres dimensiones: (1) el acervo de saberes, o la ciencia en sentido pasivo, el conocimiento acumulado en algún soporte estático, tradicionalmente en libros y bibliotecas; (2) el flujo o la ciencia en sentido activo, la producción, la innovación continua, cuya clave es el método; (3) y, finalmente, los recursos, materiales o bumanos, necesarios para la innovación. Pues poco se puede hacer si no se dispone de recursos. En definitiva, podemos visualizar la ciencia como un output, resultado de una fábrica (el método) cuyo input son los recursos disponibles.

$Y$ ese es el lento proceso de institucionalización de la ciencia, que pasa de ser la actividad aislada de pioneros, similar a la obra creativa de un escritor o un pintor, a la actual y gigantesca institucionalización de la ciencia. Si buscáramos un punto de partida a la institucionalización de la ciencia, y dejando aparte el precedente de Bacon o las Sociedades Reales, sin duda deberiamos comenzar con Humboldt y la fundación en 1809 de la primera universidad investigadora, la Universidad de Berlín, que incorpora la ciencia a la Universidad. Con la Universidad de Berlín aparece ya algo radicalmente nuevo: el trabajador a quien se paga para que investigue, en este caso un funcionario público remunerado con el objetivo de que aumente los conocimientos.

El proceso de institucionalización continúa con el desarrollo del sistema universitario alemán a lo largo del XIX, modelo para todos los demás ( $y$ asi para los Estados Unidos, Japón o la española Junta para Ampliación de Estudios). Sigue con los primeras joint ventures entre departamentos universitarios y empresas en la Alemania guillermina, en el fin/comienzo de siglo, dando lugar a la primera ( $y$ en gran medida actual) industria química, farmacéutica o eléctrica, un precedente claro e ignorado de Silicon Valley. Se traslada después a las universidades estadounidense, pautadas seguin el modelo de las germanas, a comienzos de este siglo. Y estalla definitivamente después de la Segunda Guerra Mundial al generarse lo que el Presidente Eisenhower -y, tras él, el sociólogo crítico Wright Mills-llamaron el complejo militar-industrial, la alianza durante la Guerra Fría de los intereses militares y el Pentágono, las grandes empresas de armamentos y las universidades en la producción de ciencia y tecnologia en gran escala. No olvidemos que hasta Arpanet, el origen de Internet, es también un derivado de ese complejo militar-industrial-universitario ideado por las autorida-

Lo sociedad del conociniento: El orden del cambio 
des militares para el caso en que una conflagración nuclear produjera un colapso de las comunicaciones ${ }^{10}$.

El proceso significa el paso desde la ciencia artesanal a la Gran Ciencia moderna ${ }^{I I}$, de la que el primer ejemplo podría ser el proyecto Manbatan orientado a la producción de las primeras bombas atómicas, otro derivado del complejo militar-industrial. Y que no es en ultima instancia sino la aplicación a la producción cientifica de los mismos métodos que la ciencia babia elaborado para cualquier otra producción, ya sea de objetos manufacturados o de servicios. Fabricar conocimientos como se fabrican automóviles, se produce leche, o se editan periódicos. Podemos decir que la ciencia se aplica reflexivamente a si misma, la producción científica se vuelve ella misma producción científica, y por lo tanto, rutinaria, constante $y$ sistemática. Una actividad a la que se dedican cada vez mas recursos en tiempo, dinero y mano de obra. Ya no se investiga artesanalmente sino industrialmente.

$Y$ esto significa un radical cambio de escala. Pondré un ejemplo: cuando se descubre la particula omega menos en la Phisical Review, el trabajo que lo anuncia lleva la firma de más de 50 investigadores. ¿Es eso un experimento u otra cosa distinta y nueva? Pero es un problema de escala: un experimento de física de alta energía en un ciclotrón puede costar miles de millones de pesetas, implicar a cientos o miles de técnicos, cientificos ingenieros $y$, por supuesto, gestores $y$ administradores, $y$ su preparación puede llevar meses, o incluso años. Hemos pasado pues de la técnica del técnico a la técnica de la organización y la burocracia o, lo que es lo mismo, a la técnica del asalariado. $Y$ como sociólogos, podriamos añadir que del mismo modo que Marx analizó cómo el trabajador manual habia sido expropiado de sus medios de producción de modo que, para poder producir, tenía que venderse como trabajador en el mercado; y del mismo modo que Weber amplió este análisis al trabajador de cuello blanco poniendo de manifiesto que lo mismo les habia ocurrido a los administradores, los abogados o los médicos, expropiados de sus medios de producción. Hoy, otro tanto le ocurre al científico o investigador, expropiado de sus medios de producción y que debe venderse como trabajador científico para disponer del utillaje necesario.

Pero lo importante es el resultado de esta dinámica de creciente institucionalización de la ciencia, que es, por supuesto, el crecimiento exponencial del flujo de los conocimientos en todas las direcciones. 
- En primer lugar en la producción de nuevos conocimientos. Los cálculos de Price muestran que estos se doblan cada 15 años, lo que es un ritmo endiablado, brutal, de crecimiento. La mayoría de los científicos que ha habido en la historia de la humanidad (el $90 \%$ ) están vivos y su porcentaje sobre el total crece en lugar de reducirse. El número de revistas científicas, como el de especialidades (el branching) se dobla también cada quince años. En muchas ramas de la ciencia un par de lustros, a veces uno solo, es suficiente para dejar obsoleta una formación inicial. Durante la década final del siglo XX se adquirió más conocimiento que en toda la historia previa de la humanidad. Son datos que muestran un salto cualitativo indiscutible.

- Pero también en la distribución de los conocimientos, y así, la escolarización masiva postsecundaria es una consecuencia. El caudal de conocimientos necesario para poder funcionar con eficacia social es inmenso e inevitablemente la escolarización se alarga. Hasta un $70 \%$ de jóvenes acuden en USA o Japón a la enseñanza postsecundaria, universitaria, tantos como hace un siglo acudían a la escuela o como hace 50 años cursaban el bachillerato. El porcentaje de población activa con enseñanza superior es en muchos países del arrea OCDE superior al $20 \%$ lo que significa que hay grupos numerosos que han sido sistemáticamente entrenados en la lógica de la ciencia, lógica que aplican inevitablemente a sus problemas cotidianos y ordinarios. Hoy en España hay más de 1.500.000 universitarios y más de 60.000 estudiantes de doctorado, números similares, respectivamente, al de alumnos de escuela o de universitarios hace 50 años. En la sociedad del conocimiento, la universidad es el equivalente a lo que era el bachillerato en la industrial, y el doctorado el equivalente a lo que era la universidad. La ciencia es así, progresivamente, el modo usual y ordinario de pensar, es, pues, cultura y, en muchos sitios, cultura popular de masas. Ese es, en mi opinión el sentido más profundo del término sociedad del conocimiento: que si antes la cultura era la ciencia, hoy la ciencia es la cultura dominante.

- Y finalmente, en la incidencia social de ese flujo continuo de conocimientos. Los tiempos de impregnación social de las nuevas tecnologías, de comercialización y difusión, se acortan. El teléfono necesito más de medio siglo desde su descubrimiento hasta su apli- 
cación; la radio, sólo 35 años; el radar, poco más de 15; la televisión poco más de 10; el transistor, 5 años. El lag o retraso entre la producción de una conocimiento básico y su difusión es cada vez menor, de modo que la misma distinción entre investigación básica y aplicada, entre I y D se desdibuja. En ocasiones se está esperando a que el laboratorio acabe de perfilar sus conclusiones pues la línea de producción, el envasado, la comercialización, y el marketing ya están preparados, a la espera de salir a la calle. La rapidísima difusión del teléfono móvil es una muestra más de una pauta generalizada: la producción de nuevos conocimientos está ya acoplada con una sociedad radicalmente neofóbica dispuesta y preparada para recibir ese aluvión de innovaciones.

El sociólogo Anthony Giddens ha expresado así, en un libro reciente, la brutalidad de ese ritmo de innovación:

La velocidad de los avances cientificos ...es diez veces mayor que hace veinte años. La velocidad en la divulgación de los avances y descubrimientos cientificos es treinta o cuarenta veces más rápida de lo que era hace veinte años. Actualmente el volumen de la investigación cientifica que se lleva a cabo en el mundo representa cinco veces el volumen de dicha investigación bace veinte años ${ }^{12}$.

Pero si la innovación se acelera y su incidencia es más rápida, todo ello acelera insospechadamente el ritmo de cambio social. Y no es de sorprender pues que, si la ciencia se dobla cada 15 años aproximadamente, la productividad lo haga cada 18.

Pues todos esos conocimientos, inciden cada vez más rápidamente sobre los ordenes sociales, sobre la producción, sobre la comunicación, sobre el transporte y un largo etcétera, inciden sobre la realidad cotidiana, modificando costumbres y hábitos de todo tipo. Hemos institucionalizado una máquina de producir innovación y cambio social: la ciencia.

A comienzos de siglo, un agudo observador de la realidad, Thorstein Veblen, publicaba el primer estudio sociológico de la ciencia, El lugar de la ciencia en la civilización moderna ${ }^{13}$. Y señalaba que ningún otro ideal cultural ocupa un lugar indiscutible similar en las convicciones de la bumanidad civilizada. Para afirmar con énfasis: Quasi lignum vitae in paradiso Dei, et quasi lucerna fulgoris in domo Domini, tal es el lugar de la ciencia en la civilización moderna ${ }^{14}$. La ciencia -afirmaba rotun- 
do Veblen-da su carácter a la cultura moderna ${ }^{15}$. Sus palabras han resultado proféticas de modo que la ciencia, ahora sí, sin duda, es el motor más fuerte del cambio social, la variable crucial, el Deus ex machina de las sociedades modernas.

\section{4. ¿Sociedad del conocimiento o de la información?}

Sin duda uno de los campos donde la incidencia de la ciencia ha sido más fuerte es en el área de los sistemas de almacenamiento y transmisión de la información de modo que lo que soñó el gran visionario Vannevar Bush hacia 1945 en el conocido trabajo Cómo podríamos pensar, es ya una realidad:

Toda la Enciclopedia Británica cabría, pues, en el interior de una caja de cerillas, y una biblioteca de un millón de volúmenes podría caber en una esquina de nuestro escritorio. Si desde la invención de los tipos de imprenta móviles, la raza humana ha producido un archivo total....equivalente a mil millones de libros, toda esa ingente cantidad de material, microfilmado, podría acarrearse en una furgoneta...El material para el microfilmado de la Enciclopedia Británica costará unos cinco centavos de dólar y podría ser enviado por correo por otro centavo ${ }^{16}$.

Bush se equivoco en poco. No han sido la óptica y el microfilmado sino la microelectrónica y la informática, pero el resultado es similar. Tan asombroso es ese resultado que se señala frecuentemente que el elemento que singulariza las sociedades modernas es la información, y se las denomina así, "sociedades de la información". Es comprensible el término, y al menos nos proporciona una contenido concreto alternativo al uso continuo del prefijo post (ya sea post-burgués, postindustrial post-capitalista o post-moderno), que indica que estamos más allá de algo, pero sin saber aún dónde. Pero no estoy nada convencido de que sea el correcto o, al menos, el más correcto.

Sin duda el impacto de los ordenadores y de sus conexión en redes es enorme como apreciamos simplemente por la evolución de los mercados de capitales, del ebusiness, de la informática, de Internet etcétera. No es pues de sorprender que el término sociedad de la información se haya impuesto desde su uso por vez primera en el clásico (y aún excelente) libro de Daniel Bell El advenimiento de la sociedad post-industrial en 1973. 
Pero el efecto neto global de las tecnologías de la información ha sido, justamente, facilitar el acceso a la información y reducir drásticamente su coste. Se ha calculado que si la industria aeronáutica hubiera avanzado a la velocidad que lo ha hecho la microelectrónica dispondríamos de aviones Boeing 767 a un coste de 500 dólares que darían la vuelta al mundo en 20 minutos y habrían gastado 20 litros de gasolina. El poder computacional de un dólar ha crecido por un factor de 10.000 en los últimos 20 años. Como soñara Bush, un solo CDRom contiene la Enciclopedia Británica y ya empezamos a tener bibliotecas particulares de CD-Roms. Pero además, podemos acceder a miles de bases de datos vía Internet, cuyo volumen de páginas web, actualmente estimado en medio billón, se dobla cada 100 días a una tasa aproximada del millón de páginas diarias ${ }^{17}$. La web es así como una inmensa memoria colectiva de la humanidad donde todo queda registrado ${ }^{18}$. Finalmente, la cantidad de información que recibimos, que se nos envía, queramos o no, que no nos interesa, es también inmensa. En resumen: la cantidad de información de que disponemos es enorme y creciente de modo que la unidad de información, el bit, vale cada vez menos. y el coste de trasmitirla es ya casi nulo.

Pero justamente por ello, el resultado neto es que se ha acentuado la diferencia entre información y conocimiento, haciendo que este sea mas valioso y aquella menos. El problema es, crecientemente, no acceder a la información, sino saber discriminar la información relevante de la que no lo es, separar la información del ruido. Como señalaba Bush, los logros verdaderamente significativos se pierden entre el maremágnum de lo carente de interés ${ }^{19}$. Y discriminar la relevante de lo irrelevante, la información del simple ruido, no es tarea de la información sino el conocimiento. Es más, a medida que la información vale menos y su acceso se democratiza el valor del conocimiento crece. Por ello las nuestras son, y de modo creciente, sociedades del conocimiento y no tanto de la información. La sociedad industrial pudo avanzar a lomos de fuentes de energía baratas (desde la máquina de vapor al motor de combustión), pero justamente por eso no la llamamos "sociedades de energía", sino sociedades de aquello que la energía hizo posible: la industria, la fábrica. El abaratamiento de la información lo que abre es la posibilidad de una nueva fábrica, las knowledge factories (cómo llamó a las universidades Clark Kerr), las fábricas del conocimiento, disponible para quien pueda usar de esa nueva energía que es la información. 
Por lo demás, es notablemente simplificador reducir el avance científico o su desarrollo tecnológico al campo de la informática. El descubrimiento de la mecánica cuántica por Max Planck en 1900 fue la base que permitió desarrollar células fotoeléctricas y más tarde, y combinada con la física del estado sólido, transistores, diodos y el moderno chip. Y es gracias a la mecánica cuántica y a las teorías de la relatividad descubiertas por Einstein en 1905 y 1915, que descubrimos la fisión nuclear del uranio, poder nuclear que ha marcado la segunda mitad del siglo. Y por supuesto, la biología molecular, base de la ingeniería genética, está comenzando con el descubrimiento del genoma. La miniaturización y las nanotecnologías están a la vuelta de la esquina y la incidencia de los nuevos materiales es ya ubicua ${ }^{20}$. En resumen, no debemos confundir la ciencia visible en la vida cotidiana con la totalidad de la ciencia que hoy permea toda actividad tecnológica o social. La fascinación con los ordenadores y sus redes es comprensible, pero no deja de ser el "modo usual de ver las cosas", los pre-juicios, que es siempre el modo contra el que se construye la ciencia (Durkheim), Es tal el avance de la ciencia en todos los campos que hay científicos que opinan que ya queda poco por descubrir, tesis más que discutible ${ }^{21}$

\section{La economia del conocimiento}

3 ¿Qué significa esta aceleración de la producción científica y de su incidencia social? Se señala con frecuencia que da lugar a una nueva economía, una nueva política y una nueva sociedad, como indica el justamente celebre libro del sociólogo español Manuel Castells La sociedad red"2. Y en gran medida es cierto. El economista británico Alfred Marshall señaló que mientras la naturaleza...muestra una tendencia a rendimientos decrecientes, el bombre....muestra una tendencia a rendimientos crecientes... El conocimiento es nuestra mas poderosa máquina de producción. La idea fue recogida hace años por el guru del management, Peter Drucker, en un libro sorprendentemente titulado Post-Capitalist Society ${ }^{23}$, a saber, que el recurso económico básico - los medios de producción, por usar la terminología económica- no es ya el capital, ni los recursos naturales...ni el trabajo. Es y será el conocimiento. El valor se crea por la "productividad" y la "innovación", ambas aplicaciones del conocimiento al trabajo ${ }^{24}$. Se trata de una afirmación repetida hoy por los mas acreditados organismos internacionales, como el Banco Mun- 
dial: Hoy la mayoria de las economias tecnológicamente avanzadas son ciertamente economias basadas en el conocimiento. Y viceversa, el conocimiento es la llave del desarrollo, el conocimiento es desarrollo ${ }^{25}$.

$\mathrm{Y}$ así parece, a juzgar por los datos. El Banco Mundial estima que mas de la mitad del PIB en los países de la OCDE se basa en la producción y distribución del conocimiento ${ }^{26}$. Y por eso, en EEUU hay hoy más trabajadores produciendo y distribuyendo conocimiento que produciendo y distribuyendo mercancías físicas. Y, por supuesto, eso acrecienta la inversión en conocimiento de modo que los países desarrollados invierten cerca del $20 \%$ del PIB en la producción y distribución del conocimiento: el $10 \%$ en educación formal (era menos del $2 \%$ hacia 1915 ); otro $5 \%$ invertido por los empleadores; y entre un 3 y un $5 \%$ en $\mathrm{I}+\mathrm{D}$. La inversión en conocimiento es pues la mayor en casi todos los países desarrollados. La clave del futuro está en la productividad de esas inversiones en conocimiento, algo sobre lo que, por cierto, sabemos muy poco como, en general, sobre la economía del conocimiento.

Una economía que se sustenta, no en la producción de objetos o cosas, sino claramente en la producción de ideas, intangibles, (fundamentalmente innovaciones, patentes, marcas o sistemas de organización) que funcionan como bienes públicos, no excluibles. Pues a diferencia de la vieja economía, agraria o industrial, en la que el uso que un individuo hace de un producto (una naranja o una aspiradora) excluye a los demás, los nuevos productos pueden ser utilizados por cualquiera indefinidamente. Producir la idea puede ser costosa, pero reproducirla y hacerla accesible a otro tiene un coste infinitesimal. Así, el coste de producción material de un ordenador puede ser inferior al $20 \%$ de su precio; el resto son patentes, ideas, marketing y distribución. De modo que, tras la propiedad inmueble, propia de las sociedades agrarias y que definía en ellas la riqueza, y la propiedad mueble (significativamente llamada "valores") de la sociedad industrial, entramos en la propiedad de intangibles propia de la sociedad del conocimiento, que debe ser protegida con copyright, royalties o patentes. General Motors no es ya la gran empresa del mundo; lo es Microsoft.

En todo caso, hemos entrado en una espiral retroalimentada en la que la ciencia genera mayor productividad y nuevos productos, economía, y esta invierte en ciencia. La economía produce conocimien- 
tos, y viceversa, el conocimiento es la riqueza y la función de las empresas no es otra que generar nuevos conocimientos ${ }^{27}$. Un ejemplo: los Laboratorios Bell cuentan con 24.000 empleados en 22 países, pero de ellos más de 4.000 tienen título de doctor. Los investigadores de la Bell Labs tienen el honor de haber recibido nada menos que 11 Premios Nobel. En estos laboratorios han nacido el transistor, el láser, la célula solar, la comunicación vía satélite, la telefonía móvil, el ordenador digital, la transmisión de TV a larga distancia, la grabación estereofónica, las películas de cine con sonido. Actualmente registran más de tres patentes por cada día de trabajo. ¿Es esto un laboratorio, un centro de investigación o una empresa? No lo sabemos bien, pero sí sabemos que es el sueño realizado de los visionarios de la sociedad industrial, el sueño de Bacon, Saint-Simon o Comte: una sociedad de científicosempresarios.

\section{6. $\mathrm{El}$ isomorfismo ciencia- democracia-mercado}

El triunfo de la ciencia y su importancia radical en el desarrollo de la sociedad post-industrial no debería, sin embargo, sorprender, pues ciencia, democracia y mercado son sólo tres aspectos o dimensiones del mismo orden institucional.

Efectivamente, estamos acostumbrados a pensar que las instituciones centrales del mundo moderno han sido el mercado y el Estado. Es normal pues se trata de las enseñanzas acumuladas de Marx y Weber, sumadas a la experiencia moderna. Marx nos enseñó que el desarrollo de la lógica de la mercancía, del capital, era el móvil de la sociedad industrial, una lógica que, a partir de Simmel, Lukacs y la teoría crítica, sabemos se expandió a los más diversos ámbitos (arte, literatura, periodismo, e incluso relaciones personales), de modo que cuando los teóricos de le elección racional construyen mercados matrimoniales, o de otro orden, percibimos inmediatamente la lógica expansiva de la mercancía. Y ciertamente que el modo de producción correspondiente a la sociedad moderna se basa en la producción de mercancías para el mercado y el consumo es algo irrebatible.

Como también lo es que, junto a los modos de producción, todo orden social se caracteriza por unos modos de administración o gestión que, en la sociedad moderna, se articulan alrededor de una legitimidad legal-racional. Y así, junto a la economía capitalista de mercado, 
el Estado democrático ha sido la otra gran fuerza conformadora del mundo moderno. Es más, podemos pensar la historia del siglo XX como la historia del triunfo de esas dos instituciones frente a modelos alternativos. La experiencia comunista, desde 1917 a 1989 (el "corto siglo XX" de algunos historiadores) es la crónica del fracaso tanto de una economía sin mercado como de un Estado sin democracia.

Pero es también la crónica de cómo esas dos instituciones van juntas y se necesitan mutuamente. Pues las relaciones entre el Estado democrático y la economía de mercado no son las de dos vasos comunicantes, tal que si uno crece el otro mengua, en un juego de suma cero. Más bien se trata de dos manifestaciones distintas del mismo orden institucional basado en el principio de soberanía del individuo, bien como agente económico (como productor, inversor o consumidor) o como agente político (como actor político, público o elector), de modo que la libertad en un campo no se puede desvincular de la libertad en el otro. Y así, si la experiencia demostró entre 1917 y 1989 que el intento de crear Estado democrático sin mercado conduce al despotismo político burocrático, la experiencia demuestra ahora que el intento de generar mercado sin Estado (ya sea en México o, de nuevo, en Rusia), conduce a la degeneración de ambos, a un Estado corrupto y a una economía fraudulenta. En ambos casos, democracia y economía capitalista, nos encontramos pues con "mercados" en el sentido de que sujetos soberanos y libres formulan ofertas que son o no aceptadas por sus conciudadanos, todo ello en un marco normativo de transparencia informativa y regulación jurídica. Que en uno de esos mercados todos los compradores tengan los mismos recursos (un voto igual para cada ciudadano) mientras que en el otro mercado tienen recursos distintos (según su capital) no altera sustancialmente la similitud. Pues sabemos además, al menos desde La distinción de Bourdieu, que tampoco los "capitales sociales" son homogéneos.

Pues bien, lo importante pero estos efectos es que ambos órdenes institucionales, el político y el económico, tienen su contrapartida en un tercero, del que probablemente derivan: la soberanía del ciudadano como ser pensante, la libertad de pensamiento y de expresión, una consecuencia de la Reforma protestante que, al establecer el libre examen, hizo de cada individuo sacerdote de sí mismo. Los philosophes franceses del XVIII vieron claramente la conexión fáctica existente entre libertad de expresión y la consecución de la verdad pues sólo en 
un marco democrático hay lugar para el debate y la crítica, sin la cual no es posible alcanzar consensos cognitivos. La censura inhibe la producción de conocimiento. Este es un tercer mercado, isomorfo con los anteriores, en el que sujetos cognitivamente soberanos formulan enunciados que son sometidos a crítica y debate publico. Es la estructura democrática de la ciencia según fue analizada por R. K. Merton ${ }^{28}$.

Así, economía de mercado, estado democrático y ciencia no son tres ordenes institucionales separados (aunque sî sean distintos) sino, como los tres lados de un triángulo, manifestaciones diversas de un mismo principio de soberanía del individuo que acepta o no las sugerencias que le formula un tercero. Y si la libertad política no es separable de la libertad económica, ninguna de éstas puede serlo de la libertad de expresión o de juicio, que es, más bien, sus sustento y origen. $\mathrm{Y}$ dicho queda -aunque es tema que exigiría mayor desarrollo- que en mi opinión es esa libertad fundamental, la del ciudadano como ser pensante, la base de las otras dos. Por decirlo de otro modo, no es la libertad política la que, históricamente, arrastró la libertad de la ciencia, sino al contrario, esta, como libertad de juicio y de expresión, un claro derivado de la reforma protestante, la que exige aquella. Pues tan pronto como la verdad es entendida, no como el dictum de una autoridad superior, sino como la aquiescencia personal a un argumento en virtud de sus méritos, la libertad de expresión resulta ser presupuesto de la verdad. Y, tan pronto como tenemos libertad de expresión, tenemos espacio público y democracia. $Y$, tan pronto como tenemos democracia, tenemos economía de mercado. Pero sea cual fuere el orden histórico causal, es lo cierto que el orden moderno plenamente realizado (la post-modernidad de algunos) es la imbricación creciente entre ciencia, democracia y mercado ${ }^{29}$.

\section{El triunfo de la modernidad y la rutina de la transgresión}

Pero, como señalaba al principio, lo importante no es tanto el efecto de esta u aquella tecnología, sino el efecto agregado y global de la institucionalización de la ciencia. Y si observamos así las cosas lo que encontramos es una inmensa revolución que ha exacerbado hasta sus ultimas conclusiones el viejo programa de la Ilustración. Recordemos el motto de Kant, sapere aude, atrévete a saber, osa saber, ten valor. Pues bien, esto, que era un eslogan revolucionario en la sociedad del antiguo régimen, no es ya la excepción sino la regla. Todavía durante 
los siglos XIX y buena parte de este el espíritu progresista e innovador, racionalista, presente en sectores sociales importantes, tenía su contrapartida en orientaciones conservadoras, tradicionalistas, que miraban al pasado. La neofilia acelerada de los modernizadores tenía la contrapartida, en no pocas ocasiones ganadora, en la neofobia de los tradicionalistas. Y así la "duda metódica" de la ciencia se oponía al dogmatismo de la fe y la religión, como los progresistas se oponían a los tradicionalistas, la iconoclasta vanguardia artística se oponía al arte pompier o clásico, o la moda (no sólo en los vestidos) se oponía a los hábitos de todo tipo. Los progresistas o innovadores, que miraban al futuro, hacia delante, y lo generaban con su acción, tenían la contrapartida y el freno de los tradicionalistas o conservadores, que miraban al pasado y ralentizaban el progreso ${ }^{30}$. Ese es el núcleo esencial de la historia de Europa desde el siglo XVIII a finales del XX. A mediados del siglo pasado, Jaime Balmes podía escribir:

Hombres bay que viven en lo pasado, y los hay también que viven en el porvenir. Unos y otros condenan lo presente; aquellos ensalzan lo que fue, estos lo que será; los primeros se consuelan con recuerdos, los segundos con esperanzas; al fijar sus miradas en los futuro los unos exhalan un gemido y entonan funerales endechas, los otros saludan con bimno entusiasta la aurora de un nuevo dia" ${ }^{3 t}$.

Pues bien, hoy ya no hay casi neofóbicos y el espíritu innovador e ilustrado lo abarca todo. Sorprendentemente, donde más se habla de innovación es en las reuniones de empresarios, justo el grupo que tradicionalmente era conservador; mientras que donde más se habla de conservación (del medio ambiente, de la biodiversidad o de la diversidad cultural, de las identidades, de las lenguas, incluso de las tradiciones y costumbres) es en las reuniones de progresistas, tanto que casi lo único que fusiona al poderoso movimiento anti-globalizador es esa común orientación anti-innovadora y conservadora. Los que fueron progresistas e innovadores hoy tratan de conservar mientras que quienes siempre trataron de conservar, hoy impulsan todo tipo de innovaciones. ¿Qué nos ha pasado? ¿Qué ha ocurrido para este cambio tan espectacular del sentido político de la innovación?

Lo que ha pasado es algo central: que la civilización occidental y, por extensión e imposición, todo el mundo, ha institucionalizado, no el orden y la rutina, como todas las demás civilizaciones, sino al con- 
trario, ha institucionalizado la innovación y el cambio. Como señaló Ogburn hace ya cincuenta años, el cambio social es una caracteristica de los tiempos modernos ${ }^{32}$. Pero lo que tenemos delante es un cambio radical de la naturaleza del cambio. Todas las sociedades conocidas en la larga marcha de la humanidad se asientan sobre rutinas bien conocidas y legitimadas que dan lugar a todo tipo de instituciones, una de cuyas tareas principales es justamente conservar esas rutinas para evitar que el cambio las destruya. Pues bien, las nuestras son sociedades que, paradójicamente, se asientan, no en la rutina sino en el cambio, no en la continuidad sino en la discontinuidad, que han hecho de la innovación su principio axial y su regla. La regla es, por así decirlo, que todas las reglas deben revisarse, que nada es sagrado ni seguro, que todo está sometido a crítica, a reforma, a cambio. Es la generalización de la cartesiana "duda metódica" de la ciencia, la sistemática puesta en entredicho de todo saber que fusiona el baconiano Plus Ultra con el kantiano Sapere aude. De modo que lo que más se aprecia y valora no es el espíritu conservador sino el innovador, no el respeto a la tradición sino su crítica. Sociedades, pues, que no respetan nada sino, casi podría decirse, el no respetar nada. Se diría que el espíritu del 68, el triunfo de la imaginación crítica, ha acabado ganando pero paradójicamente, no contra el capitalismo, sino gracias a él ${ }^{33}$.

Como señaló Pomian en un brillante artículo, la civilización occidental se yergue así en la única civilización conocida que se basa en la trasgresión constante, que no respeta frontera alguna. La civilización europea es la única civilización de fronteras móviles. La única en erigir la trasgresión -en el sentido etimológico de la palabraen una manera de ser, es una civilización de la trasgresión, la única conocida en la historia ${ }^{34}$. No ha respetado fronteras espaciales y la desterritorialización y expansión de Occidente desde el siglo XVI hasta cubrir el mundo entero - lo que ahora, visto desde el final, llamamos globalización - fue un primer paso que se continua en la frontera espacial.

Es una marcha obsesiva hacia adelante, como señala Bauman, en la cual la negación compulsiva es la positividad... La disfuncionalidad de la cultura moderna es su funcionalidad ${ }^{35}$, añade con frase rotunda y certera.

Pero la expansión de los conocimientos es la principal frontera a movilizar, pues es ella la palanca que permite transgredir cualquier otra frontera. Plus Ultra, siempre mas allá. La modernidad -señala

La sociedod del conocimiento: El orden del cambio 
Giddens- institucionaliza el principio de la duda radical e insiste en que todo conocimiento toma la forma de bipótesis... (que) siempre están abiertas a la revisión 36 . La ciencia es progreso, que es frontera, que es expansión.

De este modo, la incidencia acelerada del cambio social forma parte ya de nuestros hábitos adquiridos: el hábito de cambiar de hábitos. Comohaseñalado Enrique GilCalvoenun recientelibroacertadamente titulado Nacidos para cambiar, los occidentales sólo estamos acostumbrados a idolatrar las novedades desde hace poco más de cien años, pueshastaentonceselhábitodominanteeralamentarladesaparición de las tradiciones $y$ resistirse al cambio ${ }^{37}$. Y efectivamente, en las sociedades tradicionales (tanto las históricas comolas aún existentes) el ritmo de cambio social es muy lento, y puesto que la longevidad es muy baja (en torno a los treinta años), las personas duraban menos que las ciudades, las casas, los muebles y los utensilios con que vivian. Es decir, en las sociedades tradicionales las personas duraban menos (y por eso cambiaban más) que las cosas que usaban y las ciudades que habitaban). Pero la revolución industrial cambió ese estado de cosas de modo que ahora las personas empezaron a durar más (o a cambiar menos) que las cosas que usaban y las ciudades en que vivian ${ }^{38}$. Y solo ahora comienza a sentirse como una vivencia real que, como señaló Marsahll Bermann, nada es estable, todo es cambiante y mudadizo, y todo lo sólido se desvanece en el aire ${ }^{39}$.Y de este modo, el rasgo más sobresaliente de los tiempos que corren podría ser el culto que se rinde al simple becho de cambiar por cambiar: ya sea de coche, de pareja o de trabajo, como de ideologia, de religión o de programa informático... los cambios son profundos, recientes, auténticos, van a transformar nuestras vidas por completo y además no han becho más que empezar.

Hasta el lenguaje publicitario nos invita, constantemente, a ir mas allá, a atrevernos, a transgredir, y nos convence de que tenemos derecho a todo, que no debemos renunciar a nada. Veamos algún ejemplotomadoalazar:Atréveteavivirmejor(Kellogg's); Hastadonde tu quieras llegar (Peugeot); ¿Tengo pinta de renunciar al placer? (Winston). Pero es también el lenguaje del orden y, por lo tanto, del Estado, que tampoco nos invita a conformarnos y aceptar el orden o las cosas, sino al contrario, nos invita a rebelarnos e innovar. Veamos un anuncio de la Dirección General de Política de la Pequeñay Mediana Empresa: 


\section{LA INNOVACIÓN TIENE MUCHO QUE VER CONTIGO}

Si tienes una buena idea

Llénala de imaginación. Hazla diferente

Estudia el diseño y la comunicación de tu producto o de tu idea como si fuera lo más importante, porque casi siempre lo es. Usa la imaginación y la inteligencia en cada uno de los procesos, busca siempre la diferencia y sorprende con el diseño. Conseguirás que tu producto o tu idea sea más deseable que las demás... Innova.

Es una recomendación de la DIRECCIÓN GENERAL DE POLÍTICA DE LA PYME.

El Gobierno apuesta por la innovación.

Solicita la Guia Básica de innovación para la pequeña empresa en los teléfonos.....

Es como si el orden mismo nos enviara constantemente el mensaje fatal Desobedéceme, que nos hace entrar directamente en el espacio de las paradojas pragmáticas. Pues, ¿cómo obedecer un orden que me invita a desobedecerle? $\mathrm{O}$ al contrario, icómo desobedecer un mandato que me invita a hacerlo? Un mensaje que aparece lleno de guiños y sutilezas: No seas tonto; desobedece, trasgrede, sólo los niños no lo bacen. Nada más patético que el desesperado intento de esos jóvenes roqueros o artistas que, sometidos al implacable control de los ratings y el mercado, creen enviar mensajes de rebeldía o situarse en los márgenes peligrosos del orden cuando lo uno y lo otro es, desde el principio, mercancía pura.

Volvamos a Francis Bacon. En el frontispicio de la primera edición del Novum Organum aparece un grabado que representa a las dos columnas de Hércules y una nave, un galeón - símbolo de la tecnología de la época, como hoy es el transbordador espacial Columbus-que la sobrepasan. Arriba, se inscribe el moto Plus Ultra, mas allá. La tecnología como camino para transgredir toda frontera avanzando incansablemente. Siempre mas allá, sin pausa, aplicando a ese transgredir toda la disciplina, el rigor y la capacidad de trabajo, la inmensa laboriosidad que esta civilización —una más en la maravillosa historia de la humanidad, no lo olvidemos-, es capaz de movilizar. Esas ideas del XVII, atrévete a saber, mas allá, han triunfado por completo y han dejado de ser ideas críticas del orden social para ser la idea central del mismo orden.

La sociedad del conocimiento: El orden del cambio 
Fray Luis de León tenía una hermosa metáfora para entender este cambio. Hablaba de dos modos de existir y los representaba como el huerto y la nave. El huerto es la vida encerrada en un hábitat que se cultiva con esmero, sometido a ritmos repetitivos y cíclicos de modo que se sabe que se puede esperar; es el beatus ille. En el huerto, hábitos y hábitat aparecen armónicamente imbricados en una economía sostenible. Y frente al huerto, la nave, de nuevo la nave como símbolo de una vida des-territorializada, sin raíces, móvil, siempre mas allá, impulsada por la tecnología. Un hábitat cambiante y una renovación acelerada de hábitos.

¿Es la nuestra pues una sociedad postmoderna o por el contrario, una sociedad hiper-moderna? En mi opinión la respuesta es clara. Ya no hay un pensamiento tradicionalista, re-accionario, que mire al pasado, y sólo lo encontramos en las fronteras aun no integradas de la civilización occidental, allí donde la modernización ha fracasado como en algunos países musulmanes o en restos del viejo Imperio Rusosoviético. Asistimos, por el contrario, al triunfo total de la modernidad y del espíritu ilustrado. Ello desequilibra por completo la relación entre orden social y cambio social, la gran dicotomía sobre la que se construye la sociología clásica desde Comte. Recordemos: orden y progreso era su esquema. Combinar el orden el Antiguo Régimen con el progreso de la Revolución. Pero no confrontamos ya sociedades que, desde el orden, se defienden frente a los riesgos del cambio, sino sociedades asentadas sobre su propio cambio. Y aquí encontramos la clave del cambio de sentido político de la innovación: pues cuando el orden deviene el cambio, los hombres de orden se hacen innovadores y viceversa, los críticos se hacen conservadores. Apoyar el orden social moderno es, paradójicamente, promover su cambio acelerado. Por el contrario, criticar el orden social es, paradójicamente, resistir ese cambio. Y por eso hoy, quizás la mayor innovación, probablemente la única, es resistirse a ella. Esto es lo que explica que los conservadores de hoy (de culturas, lenguas, identidades, especies, biodiversidad, naturaleza) sean los herederos de los progresistas del pasado mientras los innovadores de hoy son los herederos de los conservadores de antaño. Somos todos - como decía irónicamente Woody Allen en Annie Hall-, conservadores de izquierdas o progresistas de derechas. Los ilustrados del XVIII querían ser modernos y esa voluntad ha continuado durante casi dos siglos. Nosotros no podemos no ser moder- 
nos. Somos modernos aunque no nos guste. Aunque bien pensado, nunca fuimos plenamente modernos (Latour); sólo ahora lo somos.

\section{Las paradojas de la ciencia}

Y por eso también, sólo ahora empezamos a ser conscientes de la parte mala de la modernidad, de cómo el progreso puede llegar a ser regresivo, de cómo el avance puede implicar retroceso, de cómo la razón puede ser irracional y las luces producir oscuridad. Empezamos pues a ser conscientes de las consecuencias no queridas de la ciencia y el conocimiento, y ese es el campo abonado en que hunde sus raíces el discurso post-moderno, receloso de la razón. Podemos generar una utopía y un discurso post-moderno justamente porque nuestra realidad social es ya radicalmente moderna. La ciencia no es sólo la solución de la mayoría de los problemas; comienza ella también a ser parte de algunos de esos problemas. Pues, bien pensada, esta primera paradoja, la del orden del cambio, es sólo la primera de una serie de ellas.

Intentemos desentrañarlas, pues, si la ciencia es el misterio ya desvelado de nuestro tiempo, sus paradojas tienen que ser las contradicciones esenciales, la parte mala y dinámica de la parte buena.

Segunda paradoja: el conocimiento mata la sabiduría. La ciencia es un saber sólo instrumental, nos indica cómo hacer las cosas, pero en absoluto qué hacer. La ciencia nada sabe sobre la buena vida, sobre que es lo bueno y lo malo, sobre qué amar o odiar, qué es hermoso o repugnante. La ciencia conoce mucho, pero carece por completo de otro tipo de conocimiento, absolutamente necesario para la vida, y que tradicionalmente se ha vinculado con la palabra sabiduría. Pero la ciencia carece de sabiduría. $Y$ sin embargo se autodefine $-\mathrm{y}$ es aceptada casi siempre - como único saber válido. Como ya señalara Thorstein Veblen en 1906, el sentido común moderno sostiene que la respuesta del cientifico es la única auténtica y definitiva. Cientifismo - señalaba Habermas hace pocos años- significa... la convicción de que no podemos ya comprender la ciencia como una forma de conocimiento posible, sino que, más bien, debemos identificar conocimiento y ciencia ${ }^{40}$. En esa medida, en la medida en que aceptamos, erróneamente, que la ciencia es el único saber válido, se transforma en un disolvente de todo otro saber alternativo posible, y también en disolvente de todo saber de fines, en disolvente de la sabiduría. El resultado es que cada vez sabemos más 
qué podemos hacer pero, paradójicamente, sabemos menos qué debemos hacer. Como señalaba agudamente el poeta Thomas Stearns Eliot,

¿Dónde está la sabiduria

que bemos perdido con el conocimiento?

¿Dónde está el conocimiento

que bemos perdido con la información?

Y efectivamente, el juego entre estas tres formas de cognición (sabiduría, conocimiento o ciencia, e información) expresa no pocas de las contradicciones de nuestro tiempo. La información - lo señalaba antesse dobla aproximadamente cada 100 días y nadamos en masas de información; el conocimiento, más difícil de medir, parece doblarse ahora cada 15 años. Pero la sabiduría de que disponemos no es mucho mayor de la que tenían Confucio o Sócrates y, lo que es peor, no sabemos cómo producirla. Vivimos pues anegados de información; con sólidos y eficaces conocimientos científicos, pero ayunos casi por completo de sabiduría. Salvo que redefinamos la ciencia, esta nos hace, paradójicamente, al tiempo más sabios y más ignorantes.

Tercera paradoja: ignoramos lo que ignoramos. Veamos un ejemplo. El Foro Global de Investigación sobre la Salud, fundación internacional financiada por la OMS y otras instituciones, presentó en Ginebra el Informe 10/90 sobre Investigación Sanitaria 2000. El primer dato interesante es cómo enfermedad y pobreza están vinculados: los países de rentas bajas y medias agrupan al $85 \%$ de la población y soportan el $92 \%$ de la "carga de enfermedad" (una medida de la mortalidad prematura, la incapacidad y la perdida de calidad de vida por causas patológicas) mientras que los países ricos, con el $15 \%$ de la población mundial soportan solo el $8 \%$ de esa carga.

Pero el segundo dato del Informe son los prioridades en la investigación médica: de los 13 billones de pesetas que se gastan anualmente, el $90 \%$ estudia enfermedades que causan el $10 \%$ de las muertes y solo el $10 \%$ se dedica a estudiar enfermedades que causan el $90 \%$. Así, los dos principales asesinos, la neumonía y las diarreas infecciosas, que dan cuenta del $11 \%$ de la mortalidad y la incapacidad, sólo atraen el $0,2 \%$ del dinero dedicado a investigación sanitaria. $\mathrm{La}$ obesidad o el envejecimiento, problemas típicos de países ricos, atraen, por el contrario, casi toda la investigación. Por supuesto, más del $90 \%$ de los 13 
billones de fondos de investigación están en manos de un pequeño numero de países y mas del $50 \%$ corresponden al sector privado.

Analicemos ahora este dato. Por definición, sabemos lo que sabemos, pero no lo que ignoramos. Pero que ignoremos ciertas cosas y no otras no es en absoluto casual. La producción de la ciencia no es como caminar por una vía de ferrocarril que tiene los raíles trazados, de modo que podemos avanzar más o menos, pero siempre por el mismo camino y en la misma dirección. Ni se extiende tampoco como una mancha de aceite que se expande inevitablemente a partir de un centro. La ciencia es sólo el conjunto de respuestas que damos a las preguntas concretas que nos hacemos, de modo que si las preguntas no se formulan tampoco conoceremos las respuestas. No hay conocimiento alguno sin interés previo que le de sentido ${ }^{41}$. La ciencia no es una fotografía, sino un mapa (Borges) y podemos hacer muchos mapas distintos de la misma realidad. $\mathrm{Y}$ así puede ser que muchas cosas que podríamos conocer las ignoremos mientras conocemos otras que, a lo mejor, no son tan importantes. La mejor metáfora sobre la naturaleza del conocimiento científico sigue siendo la de Kant: islotes de sabiduría en un mar de ignorancia. ¿Por qué hacemos aflorar algunos islotes y no otros? Por ello, cabe pensar en dos ciencias, igualmente científicas, pero con contenidos radicalmente distintos y que respondan a cuestiones distintas, en resumen, que nos den saber sobre cosas muy diferentes. Por ejemplo, ¿por qué la medicina se centra en la enfermedad $y$ no en la salud?

Pero la conclusión es que, en una sociedad basada en el conocimiento, orientada y movilizada por la producción industrial de conocimientos, y en la que la ciencia abre el camino, la tecnología lo pavimenta y nosotros lo recorremos, la pregunta por las prioridades en la investigación científica, la pregunta por los caminos, es la pregunta crucial. ¿Por qué se abordan ciertos programas de investigación y no otros? ¿Cómo se producen, socialmente, de facto, los programas de investigación? En resumen, ipor qué sabemos lo que sabemos y por qué ignoramos lo que ignoramos? El desarrollo de la ciencia es ciego, sometido sin duda a la exigencia del beneficio o a las prioridades políticas, pero no tenemos - ni tendremos - una ciencia que oriente la ciencia. Y mientras no tengamos una sabiduría que nos diga qué merece la pena ser sabido, puede que sepamos mucho pero puede que sepamos lo que no merece la pena ser sabido.

311

La sociedad del conocimiento: El orden del cambio 
Cuarta paradoja: no sabemos que hacer con lo que sabemos: La tercera paradoja deriva del distinto ritmo al que se desarrollan lo posible y lo deseable. Pondré otro ejemplo: la oveja Dolly. Sabemos que podemos clonar ovejas, pero también seres humanos. Ya mismo. Pero no tenemos ni idea de cuando es bueno o malo hacerlo, en qué condiciones o bajo qué supuestos. Ello es consecuencia de un lag, de un retraso estructural entre el ritmo acelerado de producción de conocimientos, de una parte, y de otra, del ritmo lento de producción de consenso social sobre cómo utilizar esos conocimientos.

La producción de cultura, de consenso moral, requiere tiempo, se genera por trial-and-error, exige discusión, debate, información. Es pues un proceso iterativo y lento. Por ello, para cuando hayamos encontrado un consenso ético acerca de cómo utilizar las técnicas de clonación la biotecnología estará ya en otra frontera y el lag continuará o incluso se habrá ampliado ${ }^{42}$. No tenemos -y estamos muy lejos de tener - una máquina de producción de cultura, de producción de consenso moral, cuya eficacia sea similar a la máquina de producción de conocimientos. Y cuando pretendemos ponerla en marcha sólo podemos hacerlo como producción científica de cultura: comités de expertos que generan más ciencia para controlar la aplicación de la ciencia. En esa medida, buena parte de la ciencia se mueve en un limbo moral, mas allá del espacio socialmente definido de lo bueno o lo rechazable, de modo que sabemos muchas cosas pero no sabemos bien qué hacer con lo que sabemos.

Quinta paradoja: no sabemos qué produce lo que sabemos: La cuarta paradoja es pues la de la iatrogenia: la ciencia como estrategia para evitar problemas genera otros problemas. El aerosol destruye la capa de ozono; los pesticidas o los fertilizantes polucionan el agua; las máquinas de producir energía generan lluvia ácida que agosta otras formas de energía naturales. La alimentación de las vacas con sofisticadas harinas animales, que las hizo caníbales, genera enfermedades de nombre impronunciable. En sociedades tan complejas como las actuales, donde cada acción personal está encadenada a todas las demás acciones de todo el mundo a través de redes de interconexión extensas y profundas, es cada vez más difícil saber cual será la consecuencia última de mi acción. Como sabía Mandeville el bien general el mal y el mal genera el bien. La misma ciencia, que nos aísla en gran medida de muchos riesgos naturales, produce otros riesgos derivados de los sis- 
temas expertos en que se plasma. Las sociedades basadas en la ciencia son - como señaló Ulrich Beck en un best seller de la sociología alemana-La sociedad del riesgot. (Risikogeselschaft), sociedades de riesgo socialmente producido.

Esta no es una peculiaridad del saber moderno. Todo conocimiento es local y limitado, y ya Merton señaló hace años que jamás podemos conocer la totalidad de las consecuencias de nuestros actos. Podemos indagar la consecuencia primera, y la segunda, y la tercera, pero a partir de la consecuencia $n$, interrumpiremos el análisis. Y sin embargo, aquel acto, sigue generando consecuencias $n+1$ indefinidamente. Lo que sí es peculiar y nuevo es que las redes de interacción de las tecnologías con la sociedad y la naturaleza forman bucles auto-referentes y la consecuencia $n+1$ no está ya alejada de la acción inicial ni en el tiempo ni en el espacio. Regresa como un boomerang y nos golpea, de modo que la aplicación sistemática de la ciencia y la tecnología en sistemas expertos que permean la vida social genera nuevas consecuencias no intencionadas que sólo la propia ciencia puede estudiar.

\section{El sueño de la razón}

El resultado de este conjunto de paradojas es que los recelos hacia la ciencia aumentan. Los datos de las encuestas de los últimos años, tanto nacionales como internacionales, son sin duda muy reveladores al mostrar una creciente desconfianza hacia la ciencia ${ }^{4+}$. Hace pocos años se les preguntó a los españoles si comerían patatas transgénicas; un $59 \%$ dijo que no. Se les preguntó entonces si comerían esas patatas si fueran mucho más baratas. Los "no" subieron al $82 \%$. iFaltaría mas! iEncima, más baratas! El estudio mostraba que más del $70 \%$ de los españoles creían que el desarrollo de la ciencia y la tecnologia traerá consigo (muchos o bastuntes) riesgos para nuestro mundo. Sólo un $14 \%$ pensaba la contrario ${ }^{45}$. La idea de riesgo empieza así a ser inseparable de la de progreso.

No debe sorprendernos, pues este recelo podemos remontarlo al mito de Frankenstein de comienzos del XIX de Mary Shelley, la traducción romántica del mito clásico del aprendiz de brujo que desata fuerzas que no puede controlar. Un científico, un médico, desea crear el hombre perfecto pero lo que produce es un monstruo. Un mito que - como el coetáneo de Nosferatu/Drácula - forma parte de la 
reacción romántico-conservadora contra la sociedad moderna y que hoy descubrimos como una metáfora de la misma sociedad moderna.

El conocido grabado de Goya nos recuerda que el sueño de la razón produce monstruos. Siempre se ha interpretado la frase more ilustrado: cuando la razón duerme emergen los monstruos. Pero la frase tiene también un sentido oculto: los monstruos pueden salir del propio delirio de la razón, de lo que ella misma sueña. La ciencia ha sido siempre la solución a todo problema; hoy, por desgracia, es ya no sólo parte de la solución, sino también parte del problema mismo pues, como señala Bauman, los problemas son creados en la resolución de problemas ${ }^{46}$. Recordemos pues, de nuevo, a Eliot: ¿qué ha sido de la sabiduría con tanto conocimiento? ¿Qué será del conocimiento con tanta información?

\section{NOTAS}

1. Conferencia pronunciada en la sesión de clausura del VII Congreso Español de Sociología, Salamanca, 22 de septiembre de 2001. Quiero agradecer a mis estudiantes de doctorado, de Madrid, Bilbao y Valencia, sus comentarios a estas páginas, que le deben más incluso de lo que estoy dispuesto a admitir.

2. A. N. Whitehead, Science and the Modern World, The Free Press, New York, 1967(e.0.1925).

3. Diamond, Jared, Armas, gémenes y acero. La sociedad bumana y sus destinos. Editorial Debate, Madrid, 1998 (e. o. 1997).

4. Gordon Childe, Man Makes Himself, The Rationalist Press, London, 1936; hay traducción en Los origenes de la civilización, Fondo de Cultura Económica, México, 1976.

5. J. Ortega y Gasset, Meditación sobre la técnica, Austral, Madrid, 1965 (e. o. 1939).

6. Como señalaba Malinowski, no existe un ansia extendida por conocer; las cosas nuevas, cual los temas europeos, les resultan francamente aburridas, la ciencia no existe como poder conductor que critica, renueva y construye. Véase B. Malinowski, Magia, ciencia y religión. Ediciones de Bolsillo, Barcelona, 1978, Pág. 36 y 37.

7. Op.cit., Päg. 73.

8. Op.cit., Päg. 73 y 85.

9. Sobre el alcance de esta idea en la historia de la ciencia puede verse la excelente monografia de Robert K. Merton, On the Shoulders of Giants, 1965.

10. No deja de ser paradójico que un sistema de comunicación creado para controlar una situación de caos haya resultado en un sistema caótico e incontrolable.

11. El término Big Science fue introducido por el pionero del análisis estadístico y sociométrico de la ciencia, Derek J. De Solla Price, en 1986, en su influyente libro Little Science, Big Science... and Beyond (Columbia University Press, New York; hay traducción en Ariel, Barcelona,1973), el primer intento de cuantificar la ciencia. Véase J.M.Sánchez Ron, La Gran Ciencia, Revista de Occidente, 142, 1993. Y A.W. Weinberg, Reflections on Big Science, The MIT Press, Mass. 1967. 


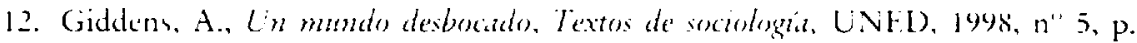
12.

13. Publicado primeramente en 1906. The Place of Science in . Modem Cicilization en American foumal of Sociologr, Xl(1906)585-609. Ampliado mas tarde con otros estudios en 1919. The Place of Scrence in Modem Civilization and Other Essays, Huesbsch, New York, 1919. Parcialmente traducido en B. Barnes, T. S. Kuhn y otros, Estudios sobre sociologia de la ciencia (Alianza Editorial, Madrid, 1980, p. 314 ss). Finalmente traducido por completo por Margarita Barañano con una introducción. Thorstein Veblen: un alegato en faror de la ciencia, puede verse en Recista Española de Investigaciones Sociológicas, 61(1993)201, por donde lo citamos.

14. Op. cit. , pag. 215.

15. Op. cit. , pag. 230.

16. Vannevar Bush, As We May Think. The Atblantic Montly. 1945; citado por la traducción al español, Cómo podriamos pensat, La Revista de Occidente, 239, 2001, p. 29. V. Bush era entonce's Director de la Oficina para la Investigación y el Desarrollo Científico del gobierno de los Estados Unidos.

17. Jose Antonio Millán. El libro de medio billón de paginas, http://jamillan.com/ ecoling.htm

18. Javier Candira, La Web como memoria organizada: el bipocampo colectivo de la Red, Revista de Occidente, 239, 2001,Pág. 87.

19. Op. cit.,Pág. 2?.

20. Véase, por ejemplo, J. M. Sánchez Ron, La ciencia del siglo XX: ciencia politica, sociedad, en F. Garcia de Cortázar, El siglo XX: mirando bacia atrás para ver bacia delunte, Papeles de FAES, n" 60. Madrid, 2001.

21. Véase Horgan, John, El final de la ciencia, 1998, y la crítica de John Maddox, What remains to be Discovered, Free Press, 1998.

22. Alianza Editorial, Madrid, 1999.

23. Pues de los muchos "post" con que se puede caracterizar las sociedades modernas esta es sin duda la menos acertada: si algo no ha cambiado es que seguimos produciendo mercancías por medio de mercancias al igual que en los tiempos de Marx. Solo que el capitalismo ha dejado de ser nacional para ser global.

24. Peter F. Drucker, Post-Capitulist Society, Butterwort-Heinemann, Oxford, 1993, Pág. 7.

25. Banco Mundial, Knoüledge for Dezelopment, Oxford University Press, 1998/99. Pág. 16 y 19.

26. Op.cit, Pág. 22.

27. Véase Nonaka. I. The Knoüledge-Creating Company, Harrard Business Review, 1991, Pag. 96. Y Nonaka, I. Y T.akeuchi. H., The knoülectge-Creating Company, Oxford University Press. New York, 1995.

28. Véase, R.K. Merton, La estructura nomutiza de la ciencia, en su compilación, Lat sociologia de la ciencia, Alianza Universidad, Madrid, 1977 (e.o. de 1942). Sobre las tesis de Merton acerca del ethos denocrático de la ciencia véase nuestro libro E. Lamo de Espinosa, J. M. González y C. Torres, La sociologita del comocimiento $y$ de la ciencia, Alianza Editorial, Madrid, 1999, Cáp. 19, redactado por Cristóbal Torres.

29. Teniendo presente, a su vez, que ciencia, democracia y mercado no son sino las manifestaciones modernas de tres invariantes o universales sociales: comunica- 
ación, parentesco y trabajo. Para ello véase mi trabajo Parentesco, trabajo y comunicat cion, presentado al Congreso de Sociología Española-2001, en prensa.

30. He analizado esta dinámica en Sociedades de cultura y sociedades de ciencia, Ediciones Nobel, Oviedo, 1996.

31. Jaime Balmes, La Sociedad, 1843, vol. I. p. 14. Reimpreso en Revista Española de Investigaciones Sociológicas, 82, 1998, pp.299 ss.

32. William F. Ogburn, La pauta del cambio social, publicado originalmente en las Actas del XIV Congreso Internacional de Sociologia, Roma, 1950; reproducido en REIS, 92, 2000, pp. 197-210.

33. Por seguir con los gurús del management, Peter Drucker, en La disciplina de la innovación. Hariard Business Reviewe, aludia al compromiso con la práctica sistemática de la innovación. Para ser innovador, hay que ser disciplinado. iLa trasgresión como nueva disciplina....!

34. Véase Krzysztof Pomian, L'Europe et ses frontiéres, Revista de Occidente, $157(1994) 25$ ss.

35. Bauman, Z., Modernidad y ambivalencia, en J. Beriain (Comp.) Las consecuencias perversas de la modemidad, Madrid, Anthropos, 1996, p. 84.

36. Giddens, A., Modemidad y autoidentidad, en J. Beriain (Comp.), Las consecuencias perversas de la modernidad, op.cit., p. 35.

37. E. Gil Calvo, Nacidos para cumbiar. Como construimos nuestras biografias, Taurus, Madrid, 2001, p. 7-8.

38. E. Gil Calvo, op.cit.,p. 10-11,

39. Marsahll Bermann, Todo lo sólido se desvanece en el aire. La experiencia de la modemidad, Editorial Siglo XXI, Madrid, 1988.

40. J. Habermas, Knowledge and Human Interests, Beacon Press, Boston, 1971, p. 4.

41. Esta es la enseñanza más importante de la sociología del conocimiento. Véase de nuevo, el texto citado de Habermas.

42. La tesis del cultural lag, del retraso entre el avance acelerado de la cultura material y el lento de la cultura inmaterial, fue desarrollada por William Ogburn en Social Change, Vicking Press, New York, 1932 (e.o., 1925).

43. Hay traducción en Paidós, Barcelona, 1996.

44. Véase Miller, Jon D, Rafael Pardo y Fujio Niwa, Percepciones del público ante la ciencia y la tecnología. Estudio comparado de la Union Europea, Estados Unidos, Japón y Canadá, Fundación BBV, Madrid, 1998.

45. Datos de Opinión, Boletín del CIS, junio, 1997, num.11, estudio 2242. Los datos eran de marzo de ese año.

46. Z. Bauman, op.cit., pág., 90. 